\title{
Realidad Virtual: Un Aporte Real para la Evaluación y el Tratamiento de Personas con Discapacidad Intelectual ${ }^{1}$
}

\section{Virtual Reality: A Real Contribution for the Evaluation and Treatment of People with Intelectual Disability}

\author{
Claudia P. Pérez-Salas \\ Pontificia Universidad Católica de Chile, Chile
}

(Rec: 16 noviembre 2007 Acep: 07 noviembre 2008)

\begin{abstract}
Resumen
La Realidad Virtual es un tipo de simulación computacional que permite recrear ambientes para que un sujeto pueda interactuar en ellos, y vivenciar esta experiencia como si ocurriera en un entorno verdadero. El presente artículo expone las principales aplicaciones de esta tecnología en el diseño de ambientes para la evaluación e intervención de personas con discapacidad intelectual, focalizándose en las utilidades que la Realidad Virtual tiene para la neuropsicología en general, y para esta población en particular. Asimismo, se analizan aspectos del diseño de ambientes y de los dispositivos de entrada de datos que podrían condicionar la efectividad de tales intervenciones, y ciertos aspectos éticos necesarios de considerar en su utilización. Finalmente, los aportes y limitaciones de la aplicación de esta tecnología en el campo de la discapacidad intelectual son ponderados y se sugieren otras áreas potenciales de desarrollo.
\end{abstract}

Palabras clave: Realidad virtual, neuropsicología, discapacidad intelectual, intervención, evaluación

\begin{abstract}
Virtual Reality is a particular computational simulation which allows recreating real environments where the subject is able to interact and live the experience as it were in a real setting. This article presents the principal applications of this technology in the design of environments for assessment and intervention of intellectually disabled persons, focusing in the advantages of Virtual Reality for neuropsychology in general and this population in particular. Moreover, environment design features and data device are analyzed as variables which could condition the intervention effectiveness. At the same time, it is remarked that some ethic aspects must be considered in the application of this technology. Finally, the contributions and limitation of the application of Virtual Reality in the intellectual disability field are considered and other areas of potential development are suggested.
\end{abstract}

Key words: Virtual reality, neuropsychology, intellectual disability, intervention, assessment.

1 Correspondencia a Claudia Paz Pérez-Salas: Programa de Doctorado, Escuela de Psicología, Pontificia Universidad Católica de Chile, Vicuña Mackenna 4860, Macul, Santiago, Chile.E mail: cpperez@uc.cl 


\section{Introducción}

En estos días aprender a utilizar el sistema de transporte para un ciudadano común puede ser un asunto bastante complejo. Esto implica no solamente la identificación del número correcto del bus, sino además una serie de comportamientos como ubicar dónde está el paradero, saber dónde y cómo pagar el pasaje y cómo solicitar que el bus se detenga en el destino deseado, entre muchos otros aspectos. Ahora imagine cuán complejo puede resultar esto para una persona con discapacidad intelectual, y cuántos ensayos y frustraciones podría acarrearle un entrenamiento in situ de esta habilidad. Actualmente ello no es necesario, y aunque le parezca ficción, para aprender ésta y otras conductas para la vida independiente, no se requieren buses, calles, ni monedas, sino sólo un mundo virtual donde tales habilidades puedan ser evaluadas, desplegadas, repetidas y perfeccionadas hasta su adquisición.

Más de 15 años han pasado ya desde que comenzaran las primeras discusiones acerca de las utilidades que tendría la aplicación de la Realidad Virtual (en adelante, RV) en la Neuropsicología ${ }^{2}$. Tales discusiones surgieron, en gran medida, como respuesta a las críticas realizadas respecto de la poca validez ecológica de sus mediciones y, por ello, su cuestionable validez predictiva en contextos naturales (Wilson, 1997). Frente a tal escenario, la RV constituía una promesa de que era factible generar ambientes simulados que contribuyeran a una evaluación y/o intervención cognitiva más sistemática y controlada, así como a actualizar y dar un giro provechoso a la neuropsicología (Johnston, 1995; Rizzo, 1994).

Desde entonces, comenzaron a desarrollarse una serie de aplicaciones neuropsicológicas de la RV en personas con diversas disfunciones del sistema nervioso central (parálisis cerebral, daño cerebral por traumatismo y trastorno de déficit atencional, entre otras), que pretendían estudiar principalmente las funciones atencionales, mnésicas, ejecutivas y/o visuoespaciales de dichos individuos (Rizzo, 2002). Los resultados de tales investigaciones, referidos especialmente a la precisión alcanzada en la manipulación de los estímulos y a la relevancia y validez de las tareas cognitivas presentadas, fueron dando crédito a las altas expectativas iniciales respecto a los potenciales usos de la RV y contribuyeron a que ésta fuera pasando, paulatinamente, de considerarse un costoso juguete a una tecnología que podría ser funcional (Rizzo, 2002).

Concordantemente con uno de los intereses más recientes de esta disciplina, a saber, el rol que los procesos cognitivos desempeñan durante la ejecución de tareas funcionalmente relevantes, como por ejemplo las actividades cotidianas (Schultheis, Himelstein \& Rizzo, 2002), una de

\footnotetext{
2 Disciplina aplicada cuyo propósito es evaluar de qué manera ciertas actividades cerebrales son expresadas en conductas observables, de modo de contribuir a una mayor comprensión de los desórdenes del sistema nervioso central, su evaluación y tratamiento (Lezak, 2004).
}

las aplicaciones más recientes de la RV han sido los ambientes simulados para el tratamiento y/o evaluación de personas con discapacidad intelectual [2] $]^{3}$. Lo anterior surge además como respuesta a la redefinición de esta discapacidad, ya no sólo como la presencia de limitaciones en el funcionamiento intelectual de un individuo, sino también por la concomitancia de deficiencias en el comportamiento adaptativo, es decir, en el conjunto de habilidades conceptuales, sociales y prácticas aprendidas por el sujeto para funcionar en su vida diaria (AAMR, 2002) ${ }^{4}$.

Los inconvenientes para abordar este constructo en personas con discapacidad intelectual por medio de los instrumentos convencionales son numerosos. Así, entre otros aspectos, cuentan la dificultad de mediciones directas (i.e., auto-reporte) y la complejidad y potenciales peligros de entrenar el comportamiento adaptativo en contextos naturales. Por ello, la RV comenzó a ser visualizada como una alternativa que podría soslayar los inconvenientes de los métodos tradicionales y constituirse, por tanto, en una vía de intervención apropiada para dichos individuos.

De este modo, comenzó a existir un mayor interés por diseñar ambientes virtuales para el entrenamiento y evaluación de destrezas cotidianas en personas con discapacidad intelectual, el cual se tradujo en un aumento de investigaciones en el tema. Si bien escasas aún, estas investigaciones han reportado resultados interesantes que hacen necesario un análisis respecto a cuáles son los aportes que la RV podría hacer en esta área, y bajo qué condiciones dichas aplicaciones serían efectivas. Lo anterior, contribuiría al desarrollo del campo y avalaría su utilización en este tipo de población, la cual por sus características de vulnerabilidad, debe ser protegida de potenciales daños derivados de tratamientos novedosos (Iacono, 2006).

Con el propósito de mostrar un panorama general de la $\mathrm{RV}$ en el área, el presente artículo comenzará con una breve descripción de las características de la RV; seguido de una revisión de las principales investigaciones que han utilizado ambientes simulados para el estudio del comportamiento adaptativo en personas con discapacidad intelectual; las utilidades que los ambientes virtuales brindan a la

\footnotetext{
En el presente artículo se acoge la recomendación realizada por la AAMR (2002) de sustituir el término "retardo mental" por el de "discapacidad intelectual" por considerarse más apropiado y menos peyorativo. Este último se define como la presencia de limitaciones significativas, tanto en el funcionamiento intelectual como en el comportamiento adaptativo del individuo y que surgen antes de que la persona alcance los 18 años de edad.

4 Las limitaciones en estas habilidades interfieren en la capacidad del individuo para desenvolverse adecuadamente en su vida cotidiana y para responder apropiadamente a las demandas ambientales. Algunos ejemplos de habilidades conceptuales lo constituyen la adquisición del lenguaje y el concepto de dinero; de habilidades sociales, la responsabilidad, seguimiento de instrucciones y autoestima; y de habilidades prácticas, las actividades de la vida diaria como preparar comidas, utilizar el transporte público y realizar compras, entre otras (AAMR, 2002).
} 
neuropsicología y cuáles son las ventajas específicas de su aplicación en este tipo de discapacidad. Luego de esto se analizará qué aspectos del diseño del ambiente virtual y de los dispositivos de entrada de datos podrían condicionar su efectividad, y qué precauciones éticas deben tenerse en cuenta en su aplicación.

\section{Realidad Virtual: Mucho más que un juego de palabras}

Existen tres condiciones imprescindibles para considerar que un sistema computacional corresponde a una RV que son: la simulación, la interacción y la percepción. La simulación corresponde a la capacidad del sistema de replicar suficientes aspectos de la realidad como para convencer al usuario de que ésta constituye una situación paralela, siendo clave para ello que las reglas que la rijan sean iguales a las de la vida real (v.g., un objeto que ha perdido su apoyo debe caer). La interacción por su parte, implica la posibilidad de tener control sobre el sistema creado y, por ende, que los cambios producidos en el mundo artificial dependan del usuario y no de una programación previa (v.g., si el usuario desea mover un objeto de lugar éste debe desplazarse). Finalmente, la percepción corresponde al elemento más definitorio de la RV y consiste en la capacidad del sistema de estimular los sentidos del usuario (o directamente el cerebros) de modo de lograr que el sujeto experimente una sensación de "inmersión" en un ambiente digital, es decir, la sensación de estar experimentando el ambiente, $\mathrm{y}$ no simplemente observándolo (v.g., sensación de estar "dentro" del ambiente) (Lavroff, 1994; Parra, García \& Santelices, 2001).

Dado lo anterior, es posible entender la RV como un tipo de simulación computacional, interactiva y tridimensional de ambientes (o situaciones) reales que propicia que el usuario se sienta inmerso en ellos, y que pese a su artificialidad, los perciba como reales gracias a la estimulación brindada a sus órganos sensoriales (Parra et al., 2001).

En función de las tres características anteriores, se han entregado diversas definiciones y clasificaciones de RV de acuerdo al énfasis dado a cada aspecto en la investigación particular que la utiliza. Es así como según el grado de inmersión existen desde los llamados sistemas de escritorio, los cuales muestran imágenes en un monitor y apelan a la imaginación del sujeto para sumergirse en una RV parcial, hasta los sistemas de inmersión prácticamente total que, por medio de sofisticados dispositivos, relacionan al usuario de manera tan estrecha con el ambiente virtual que lo aíslan en cierta medida del ambiente real (Holden, 2005; Parra et al., 2001) (ver Figura 1). Asimismo, para aumentar la sensación de presencia, especialmente en los sistemas de escritorio,

\footnotetext{
5 Los sistemas de interfaz cerebro- computador utilizan las señales cerebrales, captadas por electrodos colocados sobre el cuero cabelludo, como dispositivo de entrada o salida de datos que permite interactuar con el ambiente virtual (Suh et al., 2006).
}

es posible incluir también una representación del usuario (denominada avatar) dentro de la escena proyectada (Parra et al., 2001) o su propia imagen captada mediante cámara Web (Gesturetek, 2007).

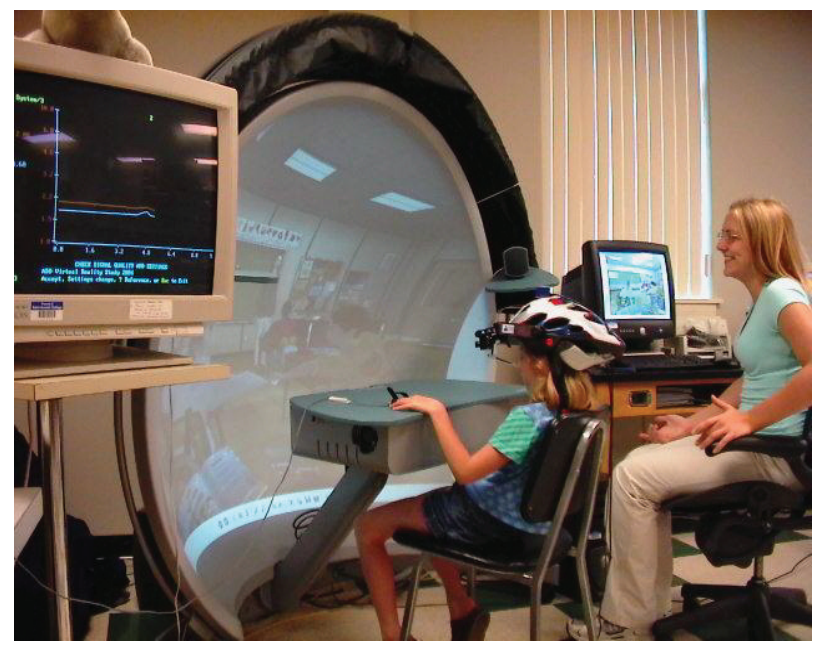

Figura 1: Niño en un ambiente virtual de alta inmersión, usando casco virtual y rastreador de posición ( $V R$ Classroom, 2007).

Respecto al tipo de ambientes virtuales creados hasta la fecha, es posible señalar que éstos han sido múltiples y han obedecido a los más diversos propósitos. A continuación se expondrán específicamente aquéllos que simulan escenarios comunes y cotidianos de la vida real (tales como una calle o un supermercado), para ejemplificar la forma en que éstos han sido utilizados en investigaciones sobre discapacidad intelectual.

\section{Ambientes virtuales para personas con discapacidad intelectual}

La utilización de ambientes simulados para el estudio de la discapacidad intelectual puede considerarse un área en desarrollo dentro de la neuropsicología, debido al limitado número de investigaciones controladas en la materia. Sin embargo, ésta ha reportado hallazgos relevantes de ser analizados de modo de ponderar sus utilidades reales así como inspirar futuros desarrollos en el campo.

De este modo, es posible apreciar que la forma principal de aplicación neuropsicológica de la RV en personas con discapacidad intelectual ha sido en el ámbito del comportamiento adaptativo, y más escasamente, se ha investigado su utilidad para intervenir y/o evaluar funciones específicas.

En este sentido, los ambientes virtuales han sido empleados mayoritariamente como métodos de entrenamiento de habilidades para la vida independiente y como formas de evaluación de la adquisición de tales habilidades. Los estudios en este ámbito han demostrado que el aprendizaje adquirido por esta vía, puede ser transferido a las situaciones 
de la vida real en donde se requiere de tales capacidades (Standen, Brown \& Cromby, 2001).

Un ejemplo de esto lo constituyen las investigaciones que utilizan el supermercado virtual para entrenar la habilidad de realizar compras, elegir artículos y ejecutar transacciones monetarias simples (Brown, Stewart \& Wilson, 1995; Cromby, Standen, Newman \& Tasker, 1996; Standen \& Cromby, 1995). En este sentido, Cromby et al. (1996), en un estudio controlado y de asignación aleatoria con 19 estudiantes con discapacidad intelectual severa, examinó si la práctica de comprar en un supermercado virtual podría tener efectos beneficiosos sobre la compra en un supermercado real (encontrar 4 ítemes en los estantes y llevarlos donde se encuentra la cajera). Los autores concluyeron que pese a que inicialmente no habían diferencias entre el grupo control y experimental, los estudiantes que fueron entrenados en el supermercado virtual fueron significativamente más rápidos y precisos en elegir los ítemes solicitados que el grupo control (mayor número de ítemes correctos en el carro de compra). Lo anterior fue explicado por los autores debido a la similitud del ambiente virtual con un supermercado real (pasillos, estantes), pero también a la simplicidad del diseño que le otorga cierto grado de flexibilidad al aprendizaje y, por ende, la expectativa en el sujeto de que puede encontrarse con tal variabilidad en las experiencias sucesivas.

Otras investigaciones sobre el uso de ambientes virtuales en el entrenamiento de habilidades para la vida cotidiana han incluido la preparación de comida en una cocina virtual, cuyos resultados indican que este tipo de entrenamiento es igual de efectivo que el realizado en una cocina real y más efectivo que la instrucción con libro de trabajo y la ausencia de tratamiento. Se ha demostrado además que esto puede ser generalizado posteriormente en entornos reales, con la ventaja de no someter a los participantes a situaciones riesgosas durante la adquisición de la habilidad (Brooks, Rose, Attree, \& Elliot-Square, 2002).

Asimismo, evaluaciones realizadas al programa "ciudad virtual", que corresponde a un ambiente simulado que reproduce el sistema de transporte, un café y un supermercado, entre otros entornos (Brown, Kerr \& Bayon, 1998), han demostrado que dichos ambientes presentan tareas representativas de la vida real y los usuarios con discapacidad intelectual son capaces de aprender algunas habilidades básicas, al menos en el corto tiempo que dura el entrenamiento. Por ejemplo, tras haber practicado en el ambiente virtual la manera correcta de colocar las monedas en el dispositivo del bus para pagar el pasaje, se repite esta destreza durante las siguientes sesiones en un bus real (Cobb, Neale \& Reynolds, 1998).

Finalmente, también se ha explorado la utilidad de los ambientes virtuales para el entrenamiento de habilidades cognitivas superiores como la toma de decisiones. Esta posibilidad ha sido escasamente investigada, pero es posible citar un estudio realizado por Standen e Ip (2002) en el cual se pretendió establecer mediante una comparación pre y post tratamiento, si la actividad en un ambiente virtual que involucrara tomar decisiones o seleccionar opciones, podría mejorar la habilidad de personas con discapacidad intelectual para decidir en otros contextos.

La primera tarea de este experimento consistió en presentar a los participantes 10 cartas con un objeto familiar impreso en uno de sus lados (v.g., manzana, pez), las cuales fueron puestas boca abajo antes de solicitar que cada individuo tomara una, escogida por el investigador. El tiempo de reacción fue medido para cada uno de los 10 intentos. La segunda tarea consistió en solicitar al participante que escogiera libremente 2 ítemes de una lista de compra de 10 elementos (dibujos), mostrados en un monitor y nuevamente fue registrado el tiempo de reacción. Finalmente, se les dijo a los participantes que uno de los ítemes ya no estaba disponible y que tenían que escoger otro (elección forzada). Luego de completar las mediciones de la línea base para la toma de decisiones, el grupo experimental pasó seis sesiones trabajando en algunas de las secciones de la "ciudad virtual" (Brown et al., 1998) antes de repetir nuevamente la evaluación de toma de decisiones. Luego de la intervención, hubo una reducción significativa en el tiempo promedio para tomar una decisión en el juego de carta y en la lista de compra respecto a la línea base. También hubo una mejora en el tiempo para escoger forzadamente alternativas en la lista de compra, aunque ésta no fue estadísticamente significativa. El grupo control en cambio no mostró mejora en ninguna de las tres mediciones.

Si bien puede considerarse como un estudio piloto y que tiene problemas tales como una muestra pequeña $(n=16)$, o que las medidas de respuesta sólo se basan en el tiempo de reacción y no en las elecciones hechas en la lista de compra, esta investigación ofrece luces respecto a otra vía de desarrollo en las aplicaciones de la RV, es decir, su utilización con fines de intervención y evaluación de funciones superiores específicas.

Los casos anteriores permiten formarse una idea respecto a los tipos de ambientes simulados que han sido creados hasta el momento para personas con discapacidad intelectual, así como los propósitos que han perseguido tales aplicaciones. A continuación se analizará cuáles son las ventajas específicas que avalan su utilización como herramienta neuropsicológica.

\section{Ventajas de la Realidad Virtual para la neuropsicología}

La RV puede contribuir a la neuropsicología, tanto en el ámbito de la evaluación como en el de la intervención. En cuanto a la evaluación, la RV puede ayudar a incrementar la confiabilidad de las mediciones, a través de la reducción del error aleatorio logrado con el mayor control ejercido sobre el ambiente experimental, y la alta estandarización 
en la administración de los estímulos e interpretación de los puntajes (Rizzo, Buckwalter \& van der Zaag, 2002; Schultheis et al., 2002). De este modo, es posible controlar incluso aspectos como la presencia de distractores durante la evaluación, los cuales pueden introducirse planificadamente de modo de evaluar su impacto en el rendimiento del sujeto durante la tarea experimental, en vez de pretender aislar o eliminar su efecto como en la mayoría de las condiciones tradicionales de evaluación (Rizzo \& Buckwalter, 1997).

Asimismo, los ambientes virtuales posibilitan realizar evaluaciones más fiables del comportamiento adaptativo de individuos que evidencian deficiencias en estas habilidades, al soslayar la necesidad de acudir a la opinión de cuidadores o familiares para acceder a este constructo, mediante tareas que permiten una evaluación más directa y sistemática de él. Dicho aspecto es relevante puesto que, según Raynes (1987), la mayoría de los instrumentos de evaluación existentes tienen serios inconvenientes para estimar su confiabilidad y validez, debido a la dificultad de considerar en un solo test el amplio rango de habilidades incluidas, al tipo de ítemes utilizados (generalmente de distinto formato), y sobre todo por la interferencia del agente externo que debe calificar el comportamiento del sujeto evaluado, siendo muchas veces la confiabilidad interjuez el máximo indicador psicométrico al que se puede aspirar.

Desde otra perspectiva, la RV puede contribuir también a incrementar la validez de las mediciones. Así, Rizzo y Buckwalter (1997) señalan que esta tecnología posibilitaría definir más específica y válidamente los constructos cognitivos en los instrumentos de evaluación basados en ella, debido a que el sistema de codificación usado en las evaluaciones por medio de RV permitirían cuantificar al mismo tiempo (y en forma paralela), varios aspectos de las respuestas del examinado, tales como estrategias de resolución de problemas, latencia de respuestas, preferencias de campo visual, entre otras. Lo anterior permitiría una delimitación más clara de la función que se desea evaluar, a diferencia de la mayoría de los sistemas de codificación usados en las pruebas tradicionales (v.g., número de símbolos marcados correctamente) que no permiten separar adecuadamente los diferentes componentes cognitivos que deben ponerse en funcionamiento para realizar una tarea.

La RV puede contribuir además a aumentar la validez ecológica de las evaluaciones debido a que el rendimiento funcional/cognitivo puede ser evaluado en un ambiente muy similar al real (Rizzo \& Buckwalter, 1997; Rizzo, Schultheis, Kerns \& Mateer, 2004) y, por ello con estímulos más relevantes y atingentes (Schultheis et al., 2002).

En cuanto a la validez predictiva, ésta podría verse favorecida con la utilización de RV, debido a que dicha tecnología permite evaluar funciones cognitivas dentro de ambientes e interacciones dinámicas. Esto es una gran ventaja puesto que, mientras las mediciones tradicionales otorgan información parcelada respecto a los componentes de funciones cognitivas y predicen potencialmente cómo ciertos déficits podrían trasladarse al mundo real, la RV permite examinar estas relaciones directamente, posibilitando la evaluación de comportamientos cognitivos complejos, como la manera en que diferentes sistemas de memoria son o no utilizados concomitantemente por los individuos durante tareas de la vida real y, por ende, contribuir a una mejor predicción de la ejecución que tendrá el examinado en su vida cotidiana (Schultheis et al., 2002).

Por otra parte, la RV como herramienta de intervención para déficits cognitivos o funcionales, podría ayudar a reducir una de las mayores limitaciones que tiene la rehabilitación en este campo, y es precisamente la separación que existe entre el enfoque reconstituyente y el funcional ${ }^{6}$. De este modo, la RV permitiría generar métodos de tratamiento sistemáticos que integren los aspectos más importantes de ambos enfoques, es decir un entrenamiento reconstituyente de componentes cognitivos, pero dentro de un ambiente ecológicamente válido y funcionalmente relevante (Rizzo et al., 2002).

Asimismo, la flexibilidad para manipular estímulos en un ambiente virtual, permite ir incrementando gradual y controladamente la dificultad de las tareas. Lo anterior posibilita una estimación más individualizada de la magnitud de los apoyos requeridos por el sujeto para lograr un mejor funcionamiento en actividades cotidianas, pudiendo entonces diseñarse un plan de tratamiento más específico y acorde a sus necesidades (Rizzo et al., 2002; Schultheis et al., 2002).

Finalmente, esta posibilidad de manipular el entorno permite además crear escenarios que no necesariamente sean idénticos a la vida real, sino que los estímulos presentados puedan ser utilizados para evaluar qué grado de información aumentada es necesario presentar, con el fin de elicitar estrategias compensatorias que mejoren el funcionamiento diario (Rizzo et al., 2002) y resulten en recomendaciones neuropsicológicas más aplicables y atingentes (Schultheis et al., 2002).

Del análisis anterior es posible apreciar que son varias las ventajas que ofrece la RV a la neuropsicología, tanto para propósitos de evaluación como de intervención. En el siguiente apartado se intentará dilucidar cuáles son los beneficios específicos que ofrece esta tecnología a personas con limitaciones cognitivas.

\footnotetext{
La aproximación reconstituyente consiste en el re-entrenamiento sistemático de ciertos componentes del procesamiento cognitivo (por ejemplo, atención o memoria) de modo de reeducar a los individuos en cómo pensar. La aproximación funcional por su parte, enfatiza el entrenamiento de comportamientos y habilidades observables, siendo su objetivo principal enseñar a los individuos cómo ejecutar ciertas actividades (Rizzo et al., 2002)
} 


\section{Beneficios de la Realidad Virtual para personas con discapacidad intelectual}

Las aplicaciones neuropsicológicas de la RV pueden ser particularmente beneficiosas para las personas con discapacidad intelectual por las siguientes razones: La primera de ellas, es que ofrece a estos individuos la oportunidad de aprender de los errores sin sufrir las consecuencias reales de ellos, aspecto que contribuiría al desarrollo de habilidades debido a que podría ayudar a realizar actividades de manera independiente sin el temor de sufrir accidentes o humillaciones (Cromby et al., 1996; Standen \& Cromby, 1995), o el miedo de irritar y cansar a otros debido a las equivocaciones cometidas (SalemDarrow, 1995).

Un segundo beneficio lo constituye la posibilidad que brinda esta tecnología para convenir las reglas y conceptos abstractos sin la utilización del lenguaje u otro sistema de símbolos, dado que las cualidades de los objetos pueden ser descubiertas por la interacción directa con ellos (Cromby et al., 1996).

Un tercer beneficio lo constituye el hecho de que la $\mathrm{RV}$ le permite a las personas con discapacidad intelectual experimentar la sensación de control sobre sus procesos de aprendizaje, lo que resulta especialmente relevante dada la tendencia al comportamiento pasivo de estos individuos (Brown et al., 1995; Pantelidis, 1993).

Finalmente, uno de los beneficios más relevantes es que la RV puede ser disfrutada por las personas con discapacidad intelectual, las cuales han demostrado gran entusiasmo y motivación durante la experiencia con esta tecnología, dando luces respecto a su importancia en la autoestima y sentido de competencia (Weiss, Pninabialik $\&$ Kizony, 2003). Este último punto es de suma relevancia, puesto que el poder motivador de esta tecnología para dichos individuos podría ser aprovechado para mantener el interés en las tareas presentadas, y por tanto, lograr en mayor medida los objetivos de evaluación y/o intervención propuestos.

Todos los beneficios expuestos ponen de manifiesto que la utilización de la RV puede ser una herramienta especialmente apropiada para personas con discapacidad intelectual. No obstante, existen aspectos que podrían dificultar su efectividad en esta población. En el siguiente apartado se expondrán algunos elementos relacionados al diseño de estos ambientes simulados y a la interfaz utilizada para interactuar con él, que deben tomarse en cuenta si se desea que las aplicaciones virtuales cumplan efectivamente con las ventajas antes prometidas.

\section{Un complejo diseño con interfaces sencillas}

Diseñar un ambiente virtual destinado para que una persona con discapacidad intelectual navegue e interactúe en él, requiere de un cuidadoso análisis respecto a qué características debe tener el programa de modo de: (a) facilitar su utilización, (b) permitir su comprensión y contribuir al aprendizaje y evaluación de la habilidad para la cual fue construido, y (c) evitar la frustración y desorientación de los usuarios. Sin embargo, un diseño perfecto desde el punto de vista de la intervención y/o evaluación no basta. Es imprescindible que además se analice el grado en que el dispositivo de entrada de datos (interfaz) resulta apropiado para estas personas, dada sus limitaciones cognitivas y, en ocasiones, sus déficits motores concomitantes.

En este sentido, Standen y Brown (2006) señalan una serie de requisitos que deben cumplir los ambientes virtuales para que su utilización por parte de personas con discapacidad intelectual sea apropiada. Dicha guía comprende los siguientes aspectos:

Comunicación: Las instrucciones para operar en el ambiente virtual deben ser breves (para que sean fácilmente recordadas) e idealmente auditivas y/o icónicas (evitando el uso de textos).

Navegación: Para facilitar la navegación, la programación debe ser tolerante y permitir un margen mayor al de la realidad para realizar algunas acciones específicas (como ingresar por una puerta desde un cierto ángulo).

Apoyo al aprendizaje: El uso de íconos y refuerzos gráficos deben ser consistentes y estar estandarizados durante todo el programa (por ejemplo, verde para "sí" y rojo para "no"); de existir cuadros de diálogo éstos deben permanecer en la pantalla un tiempo suficientemente largo de acuerdo a la habilidad del usuario. Por otra parte, el ambiente virtual debe contener suficientes claves de la vida real para permitir su reconocimiento, pero no en demasía como para distraer al usuario del objetivo de aprendizaje.

Accesibilidad: Pese a que los ambientes virtuales deben ser realistas, los diseñadores deben usar entornos que ayuden a superar barreras de aprendizaje que existen en el mundo real, elaborando ambientes que realcen aspectos claves necesarios para la comprensión.

Interacción: Para facilitar la interacción en el ambiente virtual, debe evitarse la utilización de más de un botón en el dispositivo de entrada si éste es un joystick; y en el caso del mouse restringirse al botón derecho. Lo anterior, debido a que el uso de más comandos complejiza demasiado la utilización del dispositivo.

En este último sentido, es preciso señalar que la mayoría de las aplicaciones de RV para personas con discapacidad intelectual corresponden a sistemas de escritorio, proyectados en un monitor convencional. Respecto al uso de dispositivos para controlar la navegación, se postula que el joystick limitado a dos grados de libertad es recomendable para obtener el máximo provecho (arriba/abajo o izquierda/ derecha), dado que mientras más opciones ofrezca el dispositivo, más difícil se torna su control (Lannen, Brown \& Powell, 2002). Asimismo se señala que el joystick sería 
preferible a la utilización del teclado para la navegación (Standen, Brown, Anderton \& Battersby, 2006), y en el caso de tareas de interacción, tanto el mouse como la pantalla táctil serían igualmente efectivas, aunque esta última ha demostrado ser más difícil de calibrar y operar cuando se requiere interactuar con objetos pequeños (Brown et al., 1997 citado en Lannen et al., 2002).

Sin embargo, pese a que tales dispositivos pueden ser apropiados para muchas tareas de navegación e interacción, no están exentos de problemas especialmente cuando la habilidad cognitiva del usuario es extremadamente baja o existen problemas motores adicionales a la discapacidad intelectual.

En el primer caso, algunos estudios han reportado que gran parte del tiempo de las sesiones de entrenamiento con $\mathrm{RV}$ en personas con discapacidad intelectual severa han sido utilizados en proveerles asistencia con los dispositivos de entrada (Anderton, Standen \& Avory, 2004; Standen, Brown, Horan \& Proctor, 2002). Otras investigaciones han reportado la gran dificultad que tienen aquéllos con discapacidad intelectual y motora concomitante para operar y controlar tales dispositivos (Brown, Neale, Cobb \& Reynolds, 1999).

Para dar respuesta a este problema Lannen (1997 citado en Lannen et al., 2002) desarrolló un prototipo de dispositivo de entrada de datos, llamado asiento interactivo "Mojo", el cual es económicamente accesible y posee alguno de los requerimientos necesarios para ser operado por individuos con dificultades motoras finas. Este dispositivo fue diseñado especialmente para personas con discapacidad intelectual de moderada a severa, y que presentan cierto grado de discapacidad física. Su diseño posee características similares a las de un joystick, pero permite controlar la navegación mediante el movimiento corporal del sujeto, mientras está sentado sobre él. Los resultados de su implementación señalan que su uso genera una clara disminución de la desorientación, y aunque requiere de algunos ajustes (sobre todo respecto a la calidad y tipo de interacción lograda con el sistema) podría ser útil en este tipo de población.

Otros esfuerzos por mejorar los dispositivos de entrada para este tipo de personas lo constituye el sistema Mandala ( ) Gesturetek Xtreme (GX) (Gesturetek, 2007) (Figura 2), el cual consiste en una cámara de video que captura la imagen del usuario y luego utiliza los movimientos de su cuerpo como interfaz para interactuar con el sistema. Su principal ventaja es que no necesita ningún dispositivo adicional para interactuar con el programa, lo que podría facilitar la comprensión de la tarea en individuos con limitaciones cognitivas (debido a que se evita la necesidad de aprender primero a utilizar una interfaz), aspecto que no ha sido del todo explorado aún.



Figura 2: Joven utilizando el dispositivo Mandala $\mathbb{}$ Gesture Xtreme como interfaz para tocar la batería (Drums, 2007).

\section{Consideraciones éticas}

La rápida evolución que ha tenido la $\mathrm{RV}$ y las aplicaciones exitosas realizadas en el campo de la neuropsicología, sumado a la disminución considerable que han tenido los costos de su implementación, hacen pensar que esta tecnología seguirá siendo utilizada cada vez más como herramienta de evaluación e intervención en diversas poblaciones. Por ello, es de suma relevancia que los profesionales que diseñen y apliquen ambientes simulados con dichos fines, consideren ciertos aspectos éticos que pueden estar involucrados en la utilización de esta emergente tecnología, de modo de velar por la seguridad e integridad de sus pacientes (Rizzo, Schultheis \& Rothbaum, 2003).

En efecto, existen aspectos importantes de considerar al realizar aplicaciones de RV con fines neuropsicológicos, especialmente si los destinatarios provienen de poblaciones vulnerables como los individuos con algún daño o discapacidad cognitiva (Brown et al., 1995; Rizzo et al., 2003). Dichos aspectos se refieren a los posibles efectos secundarios adversos que pueden presentarse debido a la utilización de esta tecnología; la delimitación de los criterios de inclusión para su uso; algunos asuntos de la práctica profesional involucrados; y a ciertas consideraciones respecto al impacto social del uso de los ambientes virtuales (Rizzo et al., 2003).

En cuanto a los posibles efectos secundarios adversos de la utilización de RV, se ha reportado la aparición de las llamadas "ciber- enfermedades" y "efectos posteriores". Las primeras se refieren a síntomas tales como náuseas, vómitos, desorientación, ataxia y vértigo (Kennedy, Berbaum, \& Drexler, 1994 citado en Rizzo et al., 2003), mientras que los segundos incluyen síntomas como cambios en la postura corporal, perturbaciones perceptuales- motoras, fatiga y "flaskbacks" (DiZio \& Lackner, 1992; Kennedy \& Stanney, 
1996; Rolland, Biocca, Barlow \& Kancherla, 1995). El reporte de la ocurrencia de efectos secundarios debido al uso de RV en poblaciones con alguna discapacidad varía dependiendo de factores como el tipo de programa utilizado, dispositivos técnicos escogidos, tiempo de exposición, experiencia previa con esta tecnología, entre otros (Hettinger, 1992; Regan \& Price, 1994). Sin embargo, aún no hay estadísticas claras respecto a la proporción de personas en muestras clínicas que experimentan tales efectos (Rizzo et al., 2003), lo que hace necesaria una mayor investigación respecto a la incidencia real de estos trastornos y su relación con el tipo y gravedad de la discapacidad, así como también si existen maneras efectivas de reducirlos de modo de evaluar si éstos podrían limitar la viabilidad de su aplicación en poblaciones clínicas (Rizzo et al., 2003).

Respecto a los criterios de inclusión, se postula que debe tenerse cautela en el caso de individuos con discapacidades cognitivas que tengan alteraciones de conciencia (v.g., alucinaciones, delirios o limitada autoconciencia de sus déficits), las cuales podrían provocar una mayor vulnerabilidad a presentar respuestas comportamentales negativas tras la exposición a RV (Rizzo et al., 2003). En este sentido, Standen \& Brown (2006) señalan que uno de los principales temores relacionados al uso de tecnología virtual para el tratamiento de personas con discapacidad intelectual es que dichos usuarios presenten dificultades para distinguir entre el mundo virtual y el real. Es decir, que la experiencia virtual genere expectativas poco acertadas de lo que podría suceder en el mundo real cuando se ejecutan comportamientos similares y, por ende, contribuya a que la persona que vivencia una experiencia virtual incurra en comportamientos temerarios que pongan severamente en riesgo su integridad (por ejemplo, que luego de ser atropellado por un auto virtual, crea que si atraviesa descuidadamente una calle real no habrán consecuencias importantes) (Whalley, 1993).

Al respecto Standen y Brown (2006) señalan que todos los métodos de entrenamiento en habilidades potencialmente riesgosas deben preocuparse por proteger a la persona vulnerable respecto de los peligros inherentes a ellas. Para esto es crucial la labor del tutor, más aún en el caso de las aplicaciones de RV, donde es él quien debe ir ayudando a la persona con discapacidad intelectual a hacer las conexiones y distinciones entre la realidad y el modelo virtual de ella en el cual están trabajando.

Dado lo anterior, es preciso enfatizar que los profesionales que utilicen RV como herramienta de evaluación o intervención con este tipo de poblaciones, deben realizarlo cuando esto verdaderamente constituya su campo de experticia y no como una forma de atraer a nuevos pacientes, $o$ un intento por administrar nuevos tipos de tratamientos si no están calificados para proveerlos (Rizzo et al., 2003).

Finalmente, respecto al impacto social del uso de esta tecnología, surge el temor de que su utilización en poblaciones cuya experiencia ya es de cierto grado de aislamiento social, reduzca aún más el tiempo disponible para aprender habilidades interpersonales en contextos reales (Whalley, 1993). Sin embargo, y tal como se mencionó anteriormente, la utilización de RV no debiera excluir el rol del profesional tratante, el cual debiera tener siempre presente que el ambiente virtual es un medio, y que el funcionamiento en el mundo real es la meta a alcanzar, para lo cual resulta vital la responsabilidad de éste tanto en el diseño como en la implementación de los tratamientos.

\section{Conclusiones}

La RV constituye un elemento que aporta a la neuropsicología, en cuanto permite actualizar sus instrumentos de acción por medios tecnológicos y ganar con ello mayor validez en su accionar.

De las ventajas analizadas e investigaciones aquí expuestas, es posible desprender que los mayores aportes que esta tecnología ha hecho hasta el momento en el ámbito de la discapacidad intelectual, han sido en relación al entrenamiento del comportamiento adaptativo, específicamente de las habilidades prácticas requeridas para operar en la vida diaria, tales como ejecutar conductas simples de compra, uso del transporte público y preparación de alimentos, entre otras. En esta área, la RV ha reportado ser capaz de simular ambientes ecológicamente relevantes y con un buen nivel de control experimental, donde resulta posible practicar tales destrezas y favorecer la generalización de éstas a contextos reales.

Asimismo, se desprende de la revisión teórica efectuada que los aportes en el área de la evaluación de personas con limitaciones cognitivas han estado supeditados a los alcanzados por la intervención, aplicación donde se ha utilizado la RV como forma de cuantificar el progreso respecto a las habilidades entrenadas en un ambiente simulado, pero no como un fin en sí mismo.

En este sentido, se estima que aún falta mayor desarrollo respecto a la utilidad de la RV como herramienta específica de evaluación tanto de funciones particulares como del comportamiento adaptativo, lo que podría contribuir a mejorar el diagnóstico en este tipo de población, así como planificar de mejor manera la cantidad y calidad de los apoyos necesarios para la habilitación o compensación de sus carencias.

Respecto a la tecnología utilizada, los sistemas de RV de escritorio parecen ser los más adecuados para personas con discapacidad intelectual, debido a que la inmersión en éstos no es absoluta y deja espacio a la interacción con el profesional tratante, que es probablemente lo que permite sacar el máximo de provecho a la experiencia, así como prevenir efectos adversos como la confusión respecto a la realidad y el ambiente virtual que podría llevar a estas personas a incurrir en comportamientos temerarios. 
Asimismo, de la presente revisión es posible concluir que las ventajas del uso de la RV en estos individuos, superan ampliamente a los potenciales perjuicios, al entregarles un ambiente seguro en el cual practicar habilidades que sería riesgoso o en ocasiones más costoso de entrenar en ambientes naturales. El hecho de si la RV es experimentada como una situación ficticia o genuina por las personas con limitaciones cognitivas, quienes dependiendo de su grado de discapacidad podrían no notar la diferencia, no resulta un aspecto particularmente negativo, a juicio de esta autora, si existe el apoyo de un profesional que oriente la intervención. En este sentido, es posible hipotetizar que incluso la dificultad para discernir entre virtualidad y realidad, pudiera contribuir a mejorar la validez externa de las aplicaciones realizadas por estos medios.

Dentro de las proyecciones de la aplicación de la RV en este campo, se estima que una posible área de desarrollo podría ser el diagnóstico del comportamiento funcional de aquellas personas con discapacidad motora severa concomitante a la discapacidad intelectual, donde la utilización de esta tecnología podría contribuir a una mejor distinción entre ambos déficits y con ello al diseño de planes de intervención más apropiados.

Por último, es preciso enfatizar la importancia del trabajo interdisciplinario en las aplicaciones diseñadas para la intervención de personas con discapacidad intelectual, donde no sólo se requiere de la experiencia del psicólogo, sino también de los educadores especiales, terapeutas ocupacionales, diseñadores gráficos, ingenieros informáticos y computacionales, de modo de ir generando herramientas cada vez más apropiadas y atingentes a las necesidades de estos individuos.

\section{Referencias}

Anderton, N., Standen, P. J. \& Avory, K. (2004). Using switch controlled software with people with profound disabilities. En P.M. Sharkey, R. McCrindle \& D. Brown (Eds.), Proceedings of the fifth international conference on disability, virtual reality and associated technologies (pp. 269-274). Oxford: New College.

American Association of Mental Retardation [AAMR]. (2002). Definition of Mental Retardation. Extraído el 1 de Junio, 2007: http://www.aaidd. org/Policies/faq mental_retardation.shtml

Brooks, B. M., Rose, F. D., Attree, E. A. \& Elliot- Square, A. (2002). An evaluation of the efficacy of training people with learning disabilities in a virtual environment. Disability and Rehabilitation, 24(11/12), 622-626.

Brown, D. J., Kerr, S. J. \& Bayon, V. (1998). The development of the Virtual City: A user centred approach. En: P.M. Sharkey, R. McCrindle $\&$ D. Brown (Eds.), Proceedings of the 2nd European Conference on Disability, Virtual Reality and Associated Techniques (pp. 11- 15). Reading, UK: University of Reading.

Brown, D. J., Neale, H., Cobb, S. V. \& Reynolds, H. (1999). The development and evaluation of the virtual city. International Journal of Virtual Reality, 4(1), 28-41.

Brown, D. J., Stewart, D. S. \& Wilson, J. R. (1995). Ethical pathways to virtual learning. In Proceedings of Conference on Virtual Reality and Persons with Disabilities. Northridge, CA: California State Universi- ty. Extraído el 28 Mayo, 2007: http://www.csun.edu/cod/conf/1995/ proceedings $/ 0010$. htm

Cobb, S. V. G., Neale, H. R. \& Reynolds, H. (1998). Evaluation of virtual learning environments. Proceedings of the 2nd European Conference on Disability, Virtual Reality and Associated. Techniques (pp. 17-23). Reading, UK: University of Reading.

Cromby, J., Standen, P., Newman, J. \& Tasker, H. (1996). Successful transfer to the real world of skills practiced in a virtual environment by students with severe learning disabilities. En Proceedings of the $1^{\text {st }}$ European Conference on Disability, Virtual Reality and Associated Technologies (pp. 103- 107)._Reading, UK: University of Reading.

DiZio, P. \& Lackner, J. R. (1992). Spatial orientation, adaptation, and motion sickness in real and virtual environments. Presence: Teleoperators and Virtual Environments, 1, 323.

Drums [Fotografia]. (2007). Extraído el 14 Noviembre, 2007: http://www. gesturetek.com/images/irex/titles/drums.jpg

Gesturetek (2007). Product Solution: Gesturetekxtreme software. Extraído el 28 Mayo, 2007: http://www.gesturetek.com/gestxtreme/gestxtreme_software.php

Hettinger, L. J. (1992). Visually induced motion sickness in virtual environments. Presence: Teleoperators and Virtual Environments. ${ }_{ \pm}$ 306-307.

Holden, M. K. (2005). Virtual Enviroments for motor rehabilitation: Review. Ciberpsychology \& Behavior 8(3), 187- 211.

Iacono, T. (2006). Ethical challenges and complexities of including people with intellectual disability as participants in research. Journal of Intellectual \& Developmental Disability, 31(3), 173- 179.

Johnston, R. (1995). Is it live or is it memorized? Virtual Reality Special Report, 2, 53-56.

Kennedy, R. S. \& Stanney, K. M. (1996). Postural instability induced by virtual reality exposure: Development of certification protocol. International Journal of Human-Computer Interaction, 8(1), 2547.

Lannen, T., Brown, D. \& Powell, H. (2002). Control of virtual environments for young people with learning difficulties. Disability and Rehabilitation, 24(11/12), 578-586.

Lavroff, N. (1994). Mundos virtuales, realidad virtual y ciberespacio. México: Instituto Tecnológico y de Estudios Superiores de Monterrey.

Lezak, M. D. (2004). Neuropsychological assessment_(4 ${ }^{\text {a }}$ Ed.). Oxford: Oxford University Press.

Pantelidis, V. S. (1993). Virtual reality in the classroom. Educational Technology, 33(4), 23-27.

Parra, J. C., García, R. \& Santelices, I. (2001). Introducción práctica a la realidad virtual. Concepción: Ediciones Universidad del Bío Bío.

Raynes, N. V. (1987). Adaptative behaviors scales. En: J. Hogg \& N. V. Raynes (1987). Assessment in mental handicap. A guide to assesment practices, test and checklists (pp. 81-106). Croom Helm: Cambridge.

Regan, E. \& Price, K. R. (1994). The frequency of occurrence and severity of side-effects of immersion virtual reality. Aviation, Space, \& Environmental Medicine, 65, 527-530.

Rizzo, A. A. (1994). Virtual reality applications for the cognitive rehabilitation of persons with traumatic head injuries. En: H. J. Murphy (Ed.), Proceedings of the 2nd International Conference on Virtual Reality And Persons With Disabilities. Northridge, CA: California State University. Extraído el 28 Mayo, 2007: http://www.csun.edu/cod/conf/1994/ proceedings/Thi $\sim 1$.htm

Rizzo, A. A. (2002). Virtual reality and disability: Emergence and challenge. Disability and Rehabilitation, 24(11/12), 567- 569.

Rizzo, A. A. \& Buckwalter, J G. (1997). Virtual reality and cognitive assessment and rehabilitation: The State of the Art. En: G. Riva (Ed.), Psycho- neuro- physiological assessment and rehabilitation in virtual environments: cognitive, clinical and human factors in advanced human computer interactions (pp. 123- 146). Amsterdam: IOS Press.

Rizzo, A. A., Buckwalter, J. G. \& van der Zaag, C. (2002). Virtual environment applications in clinical neuropsychology. E: K. Stanney (Ed.), Handbook of virtual environments (pp. 1027-1064). New York: L.A. Erlbaum. 
Rizzo, A. A., Schultheis, M., Kerns, K. A. \& Mateer, C. (2004). Analysis of assets for virtual reality applications in neuropsychology. Neuropsychological Rehabilitation, 14 (1/2), 207- 239.

Rizzo, A., Schultheis, M. T. \& Rothbaum, B. O. (2003). Ethical issues for the use of virtual reality in the psychological sciencies. En: S.S. Bush \& M. L. Drexler (Eds.), Ethical issues in clinical neuropsychology (pp. 243-280). Lisse, The Neaderlands: Swets \& Zeitlinger Publishers.

Rolland, J.P., Biocca, F.A., Barlow, T. \& Kancherla, A. (1995). Quantification of adaptation to virtual-eye location in see-thru head-mounted displays. Proceedings of the IEEE Virtual Reality Annual International Symposium '95 (pp. 55 - 66). Los Alamitos, CA.: IEEE Computer Society Press.

Salem-Darrow, M. (1995). Virtual Reality's increasing potential for meeting needs of person with disabilities: What about cognitive impairments? En: Proceedings of the $3^{\text {rdt }}$ International Conference on Virtual Reality and persons with disabilities. Northridge, C.A: California State University. Extraído el 28 Mayo, 2007: http://www.csun.edu/cod/ conf/1995/proceedings/0023.htm

Schultheis, M. T., Himelstein, J. \& Rizzo, A. A. (2002). Virtual reality and neuropsychology upgrading the current tools. Journal of Head Trauma Rehabilitation, 17(5), 378- 394.

Standen, P. J. \& Brown, D. J. (2006). Virtual Reality and its role in removing barriers that turn cognitive impairments into intellectual disability. Virtual Reality, 10, 241-252.

Standen, P. J., Brown, D. J., Anderton, B. A. \& Battersby, S. (2006). Systematic evaluation of current control device used by people with intellectual disabilities in non- inmersive virtual environment. CyberPsychology \& Behavior, 9(5), 608- 613.

Standen, P. J., Brown, D. J. \& Cromby, J. J. (2001). The effective use of virtual environments in the education and rehabilitation of students with intellectual disabilities. British Journal of Educational Technology, 32(3), 289-299.
Standen, P. J., Brown, D. J., Horan, M. \& Proctor, T. (2002). How tutors assist adults with learning disabilities to use virtual environments. Disability and Rehabilitation, 24(11-12), 570-577.

Standen, P. J. \& Cromby, J. J. (1995). Can students with developmental disability use virtual reality to learn skills which will transfer to the real world? En: Proceedings of Conference on Virtual Reality and Persons with Disabilities. Extraído el 28 Mayo, 2007: http://www.csun.edu/cod/ conf/1995/proceedings/0011.htm

Standen, P. J. \& Ip, W. M. D. (2002). An evaluation of the use of virtual environments in improving choice reaction time in people with severe intellectual disabilities. En P. M. Sharkey, C. Sik Lányi, P. J. Standen (Eds.), Proceedings of the 4th International Conference on Disability, Virtual Reality and Associated Technologies (pp. 19-24). Reading, UK: University of Reading.

Suh, D., Sang Cho, H., Goo, J., Park, K. S. \& Hahn, M. (2006). Virtual navigation system for the disabled by motor imagery. En: K. Elleity, T. Sobh, A. Mahmood, M. Iskander \& M. Karim, (Eds.), Advances in Computer, Information and Systems Sciences, and Engineering (pp. 143-148). Netherlands: Springer.

VR Classroom [Fotografía]. (2007). Extraído el 1 Mayo, 2007 del Sitio Web de University of Southern California: http://vrpsych.ict.usc.edu/ images/i58.jpg

Whalley, L. J. (1993). Ethical issues in the application of virtual reality to the treatment of mental disorders. En: R. A. Earnshaw, M. A. Gigante \& Jones, H. (Eds.), Virtual reality systems (pp. 273- 287). London: Academic Press.

Weiss, P. L., Pninabialik, B. A. \& Kizony, R. (2003). Virtual Reality provides leisure time opportunities for young adults with physical and intellectual disabilities. CyberPsychology \& Behavior, 6(3), 335- 342.

Wilson, B. A. (1997). Cognitive rehabilitation: How it is and how it might be. Journal of the International Neuropsychological Society, 3. 487-496. 